\title{
$\begin{array}{ll}\text { Research Square } & \text { Preprints are preliminary reports that have not undergone peer review. } \\ \text { They should not be considered conclusive, used to inform clinical practice, } \\ \text { or referenced by the media as validated information. }\end{array}$
}

\section{Diagnostic and prognostic value of plasma adrenomedullin compared to other biomarkers using Sepsis-3 definitions for sepsis and septic shock}

Tetsu Yonaha ( $\nabla$ tetsu.yonaha@gmail.com )

University of Miyazaki https://orcid.org/0000-0002-4935-2299

Toyoaki Maruta

University of Miyazaki

Go Otao

University of Miyazaki

Koji Igarashi

TOSOH Corporation

\section{Sayaka Nagata}

University of Miyazaki

Takeshi Yano

University of Miyazaki

Masahiko Taniguchi

University of Miyazaki

\section{Kazuo Kitamura}

University of Miyazaki

Isao Tsuneyoshi

University of Miyazaki

\section{Research}

Keywords: Adrenomedullin, Biomarkers, ICU, Sepsis, Sepsis-3, Septic Shock

Posted Date: January 2nd, 2020

DOI: https://doi.org/10.21203/rs.2.19873/v1

License: (c) (i) This work is licensed under a Creative Commons Attribution 4.0 International License. Read Full License 


\section{Abstract}

\section{Background}

Sepsis-3, the recent sepsis definitions, was modified based on a scoring system focused on organ failure; however, it would remain a time-consuming process to detect septic patients using these definitions. Adrenomedullin (AM) is a biomarker for diagnosing sepsis and septic shock, monitoring treatment efficacy, and prognosis. We conducted a study to assess the accuracy of AM for diagnosing and prognosing sepsis and septic shock based on the Sepsis-3 definitions.

Methods

This is a prospective observational single-center study. Patients admitted to the intensive care unit (ICU) were retrospectively categorized as non-sepsis, sepsis, or septic shock by Sepsis-3 definitions. Total AM (tAM) and mature AM (mAM) were measured upon ICU admission. Receiver operating characteristics (ROC) analyses were performed by calculating the area under the curve (AUC) for diagnosis and prognosis of sepsis and septic shock.

Results

A total of 98 patients were enrolled in the final analysis. Among these, 42, 22, and 34 patients were assigned to nonsepsis, sepsis, and septic shock, respectively. tAM and mAM levels significantly increased according to the severity of sepsis. The AUCs of tAM/mAM for diagnosing sepsis and septic shock were $0.879 / 0.848$ and $0.858 / 0.830$, respectively, whereas those of procalcitonin (PCT)/presepsin (PSEP) were $0.822 / 0.682$ and $0.811 / 0.661$, respectively. The AUCs of tAM/mAM on Day 1 and 3 for predicting 28-day mortality of septic patients were $0.669 / 0.5741$ and $0.931 / 0.892$, respectively, whereas those of sequential organ failure assessment (SOFA) score/lactate were 0.669/0.824 and $0.922 / 0.794$, respectively.

\section{Conclusions}

Both tAM and mAM are reliable, early biomarkers to diagnose sepsis and septic shock according to the Sepsis-3 definitions, and are comparable to PCT. Furthermore, AM level on Day 3 is a reliable biomarker to predict 28-day mortality due to sepsis, which is comparable to that of the SOFA score and lactate level.

\section{Background}

Sepsis is a common clinical condition, characterized as a systemic microbial infection and subsequent organ failure often leading to death [1-3]. Despite the development of various diagnostic and therapeutic modalities, sepsis- and septic shock-associated mortality remains high [1-3]. In-hospital mortality from septic shock is $20-50 \%$ [1-3], although mortality rates vary depending on the expertise and experience of each medical center. According to the most recent Center for Disease Control (CDC) report, it is estimated that sepsis affects approximately 1.7 million individuals in the United States annually, causing the death of 270,000 individuals, and 1 out of every 3 hospital deaths [4]. Sepsis should be diagnosed as soon as possible to potentiate the effects of early evidence-based treatment and intensive care, which can improve survival and decrease in-hospital mortality rates [5]; however, clinical and laboratory findings for sepsis are non-specific and bacterial culture results are not readily available, so sepsis diagnosis and risk stratification of septic patients is often delayed [6]. Biomarkers such as $\mathrm{C}$ reactive protein (CRP) and procalcitonin (PCT) are widely used to differentiate between systemic inflammatory response syndrome (SIRS), sepsis, and septic shock, as well as for patient risk stratification [7, 8]. Accordingly, characterizing new diagnostic biomarkers to identify patients in early stages of sepsis or septic shock is essential, as mortality rates due to septic shock are still unacceptably high despite modern intensive care medicine. 
Adrenomedullin (AM) is a potent vasodilator peptide originally identified in tissue extracts of human pheochromocytoma [9]. In the present day, AM is known to be produced in several organs and tissues [10]. It has various physiological effects on the cardiovascular system, renal function, and central nervous system, including the regulation of blood pressure and vascular tone, increasing cardiac output, promoting diuresis and natriuresis, inhibiting aldosterone secretion, and suppressing fluid intake [9-13]. Blood AM levels increase in patients with heart failure, myocardial infarction, pulmonary hypertension, SIRS, inflammatory bowel diseases, renal failure, and sepsis [10,15-18]. For sepsis, bioactive mature forms of AM (mAM or bio-AM) and the mid-region of AM (MR-proADM or proADM) are closely associated with sequential organ failure assessment (SOFA) or acute physiology and chronic health evaluation (APACHE) II scores, and can be useful as diagnostic and prognostic biomarkers [19-28]. These studies, however, were based on the Sepsis-1 or Sepsis-2 definitions. Thus, we conducted this study to assess the diagnostic and prognostic value of AM for sepsis and septic shock using the Sepsis-3 definitions, which define sepsis as infection and organ failure [29].

\section{Methods}

\section{Study design}

This prospective, observational, single-center study was conducted in accordance with the principles of the Declaration of Helsinki and was approved by the Hospital Ethical Committee for Human Studies (Ref: 0-0317) on 1 December 2014, and retrospectively registered with the Japanese Clinical Trial Registry “UMIN-CTR” (Ref: UMIN000036474). General informed consent was obtained from all short-term observational patients and written informed consent was obtained from all long-term observational patients (age $\geq 20$ years). Strengthening the Reporting of Observational Studies in Epidemiology (STROBE) guidelines were followed to prepare this manuscript. For analysis, patients were classified as non-sepsis, sepsis, or septic shock according to the Sepsis-3 definitions; sepsis was defined as a SOFA score of 2 or more following infection, and septic shock was defined as a mean atrial pressure (MAP) $<65 \mathrm{mmHg}$ or lactate concentration > $2 \mathrm{mmol} / \mathrm{L}$, despite fluid resuscitation with $\geq 30 \mathrm{~mL} / \mathrm{kg}$ crystalloid or $5 \%$ albumin, in patients diagnosed with sepsis [29]. Exclusion criteria were age $<20$ years, pregnancy, trauma, postoperative patients, or lack of informed consent.

In the short-term observational patients, residual artery blood gas samples, which were obtained close to the time of admission; and 6 hours, 12 hours, 18 hours, and 24 hours post-admission to the ICU; were used to measure AM, to eliminate additional blood sample collection and reduce the burden on patients. In long-term observational patients, blood samples were collected at the time of consent and on Days 3, 5, and 7.

\section{Data collection}

Clinical data obtained from electronic medical records included demographics, diagnosis, chronic comorbidities, laboratory test results, microbiology, and biomarker levels. The SOFA score, which consisted of six organ-specific scoring items including the respiratory system, cardiovascular system, liver, coagulation, and kidneys, was calculated daily. All blood samples except for blood culture specimens were collected from an arterial catheter. Either or both PCT and presepsin (PSEP) were measured upon admission.

\section{Biomarker measurements}

AM derives from AM precursor in a two-step enzymatic reaction. First, 185-amino acid AM precursors, known as preproadrenomedullin, are converted to glycine-extended AM, which are 53-amino acid, inactive, intermediate form of AM (iAM). Subsequently, by enzymatic amidation, iAM are converted to bioactive mature AM (mAM), 52-amino acid peptide with an amide group at the C-terminus [30,31]. Although both iAM and mAM forms circulate in the bloodstream, over $85 \%$ of total plasma AM is inactive iAM. Comparative radioimmunoassays used in previous studies could not distinguish between iAM and mAM; hence, AM sometimes indicated total AM (tAM = iAM + mAM). In our study, plasma levels of both mAM and tAM were measured by a specific fluorescence immunoassay (Tosoh Corporation, Tokyo, Japan) with two 
independent antibodies: one that bound to the ringed structure for the TAM assay and the other that bound to the middle region between the ring and the C-terminal portion of the peptide for the mAM assay, as previously described [32, 33]. Plasma PSEP level was measured with a rapid chemiluminescent enzyme immunoassay (CLEIA) using a PATHFAST immunoanalyzer (Mitsubishi Chemical Medience, Tokyo, Japan).

Additionally, we obtained data on PCT, white blood cell (WBC) count, C-reactive protein (CRP), platelets, creatinine, and prothrombin time-international normalized ratio (PT-INR), which were routinely measured at the central laboratory.

\section{Statistical Analysis}

Statistical analysis was performed using MedCalc 17 (MedCalc Software bvba, Ostend, Belgium). Data were expressed as the median (IQR: interquartile range) or with exact numbers. Continuous variables were compared using MannWhitney U or Kruskal-Wallis tests as appropriate. Receiver operating characteristic (ROC) analysis and comparison of the area under the curve (AUC) were performed to evaluate the predictability of biomarkers to diagnose and prognose sepsis and septic shock. Retrospective power analysis was performed by G*Power (Version 3.1.9.3, Dusseldorf University, Dusseldorf, Germany) software [34]. $\mathrm{P}<0.05$ was considered statistically significant.

\section{Results}

\section{Patient characteristics}


Table 1

Clinical characteristics of patients admitted to ICU by Sepsis-3 classification

\begin{tabular}{|c|c|c|c|c|c|c|c|c|c|}
\hline & \multicolumn{3}{|c|}{ All $(n=98)$} & \multicolumn{3}{|c|}{ Short-term monitoring $(n=55)$} & \multicolumn{3}{|c|}{$\begin{array}{l}\text { Long-term monitoring }(n= \\
\text { 43) }\end{array}$} \\
\hline & $\begin{array}{l}\text { Non- } \\
\text { sepsis } \\
(n=42)\end{array}$ & $\begin{array}{l}\text { Sepsis } \\
(n=22)\end{array}$ & $\begin{array}{l}\text { Septic } \\
\text { shock } \\
(n=34)\end{array}$ & $\begin{array}{l}\text { Non- } \\
\text { sepsis } \\
(n=25)\end{array}$ & $\begin{array}{l}\text { Sepsis } \\
(n=12)\end{array}$ & $\begin{array}{l}\text { Septic } \\
\text { shock } \\
(n=18)\end{array}$ & $\begin{array}{l}\text { Non- } \\
\text { sepsis } \\
(n=17)\end{array}$ & $\begin{array}{l}\text { Sepsis } \\
(n=10)\end{array}$ & $\begin{array}{l}\text { Septic } \\
\text { shock } \\
(n= \\
16)\end{array}$ \\
\hline $\begin{array}{l}\text { Age (y), } \\
\text { median } \\
\text { (IQR) }\end{array}$ & $\begin{array}{l}66.5 \\
(56.3- \\
76.0)\end{array}$ & $\begin{array}{l}66.5 \\
(52.5- \\
76.0)\end{array}$ & $\begin{array}{l}72.5 \\
(60.0- \\
79.8)\end{array}$ & $\begin{array}{l}68.0 \\
(61.0- \\
76.0)\end{array}$ & $\begin{array}{l}67.0 \\
(49.3- \\
74.0)\end{array}$ & $\begin{array}{l}67.5 \\
(55.8- \\
80.5)\end{array}$ & $\begin{array}{l}63.0 \\
(54.0- \\
75.0)\end{array}$ & $\begin{array}{l}66.5(59.5- \\
77.5)\end{array}$ & $\begin{array}{l}73.5 \\
(66.5- \\
77.8)\end{array}$ \\
\hline Male, n (\%) & $32(76)$ & $14(64)$ & $23(68)$ & $18(72)$ & $6(50)$ & $11(61)$ & $14(82)$ & $8(80)$ & $\begin{array}{l}12 \\
(75)\end{array}$ \\
\hline \multicolumn{10}{|l|}{$\begin{array}{l}\text { Diagnosis, n } \\
(\%)\end{array}$} \\
\hline $\begin{array}{l}\text { Abdominal } \\
\text { Disorder }\end{array}$ & $8(19)$ & $4(18)$ & $12(35)$ & $3(12)$ & $2(17)$ & $6(33)$ & $5(29)$ & $2(20)$ & $6(38)$ \\
\hline Abscess & $1(2)$ & $0(0)$ & $2(6)$ & $0(0)$ & $0(0)$ & $1(6)$ & $1(6)$ & $0(0)$ & $1(6)$ \\
\hline $\begin{array}{l}\text { Aortic } \\
\text { Disorder }\end{array}$ & $3(7)$ & $0(0)$ & $0(0)$ & $3(12)$ & $0(0)$ & $0(0)$ & $0(0)$ & $0(0)$ & $0(0)$ \\
\hline $\begin{array}{l}\text { Blood } \\
\text { Stream } \\
\text { Infection }\end{array}$ & $0(0)$ & $4(18)$ & $5(15)$ & $0(0)$ & $3(25)$ & $2(11)$ & $0(0)$ & $1(10)$ & $3(19)$ \\
\hline $\begin{array}{l}\text { Heart } \\
\text { Failure }\end{array}$ & $10(24)$ & $0(0)$ & $0(0)$ & $9(36)$ & $0(0)$ & $0(0)$ & $1(6)$ & $0(0)$ & $0(0)$ \\
\hline $\begin{array}{l}\text { Neurological } \\
\text { Disorder }\end{array}$ & $1(2)$ & $1(5)$ & $1(3)$ & $1(4)$ & $1(8)$ & $0(0)$ & $0(0)$ & $0(0)$ & $1(6)$ \\
\hline $\begin{array}{l}\text { Respiratory } \\
\text { Disorder }\end{array}$ & $8(19)$ & $8(36)$ & $7(21)$ & $3(12)$ & $3(25)$ & $4(22)$ & $5(29)$ & $5(50)$ & $3(19)$ \\
\hline $\begin{array}{l}\text { Skin/Soft } \\
\text { tissue } \\
\text { Disorder }\end{array}$ & $3(7)$ & $1(5)$ & $2(6)$ & $2(8)$ & $1(8)$ & $2(11)$ & $1(6)$ & $0(0)$ & $0(0)$ \\
\hline $\begin{array}{l}\text { Urinary } \\
\text { Disorder }\end{array}$ & $3(7)$ & $2(9)$ & $3(9)$ & $2(8)$ & $1(8)$ & $1(6)$ & $2(12)$ & $1(10)$ & $2(13)$ \\
\hline Others & $5(12)$ & $2(9)$ & $2(6)$ & $3(12)$ & $1(8)$ & $2(11)$ & $2(12)$ & $1(10)$ & $0(0)$ \\
\hline $\begin{array}{l}\text { 28-day } \\
\text { mortality, n } \\
(\%)\end{array}$ & $6(14.3)$ & $2(9.1)$ & $\begin{array}{l}15 \\
(45.5)\end{array}$ & $3(12.0)$ & $2(16.7)$ & 7 (38.9) & $3(17.6)$ & $0(0)$ & $\begin{array}{l}8 \\
(53.3)\end{array}$ \\
\hline
\end{tabular}

Between January 2015 and November 2018, 98 patients were enrolled in this study (Fig. 1), 43 consecutive patients from January 2015 to August 2017 were included in the long-term observational group, and 55 consecutive patients from September 2017 to November 2018 were included in the short-term observational group. There were 22 and 34 patients diagnosed by Sepsis-3 definitions with sepsis and septic shock, respectively, and 42 diagnosed as non-sepsis. Clinical 
characteristics are shown in Table 1. The abdomen and respiratory tract were the most common infection sites, whereas heart failure was the most common reason for ICU admission in non-sepsis patients. We could not track the outcome of one septic shock patient in the long-term observational group, because the patient changed hospitals. The overall 28-day mortality was $23.7 \%$ (Fig. 1 and Table 1). The 28-day mortality due to sepsis and septic shock was $30.4 \%$ (sepsis: $9.1 \%$, septic shock: $45.5 \%)$.

Table 2

Clinical parameters of patients at ICU admission

\begin{tabular}{|c|c|c|c|c|c|c|c|c|c|c|}
\hline \multirow{3}{*}{$\begin{array}{l}\text { Clinical } \\
\text { parameters }\end{array}$} & \multicolumn{3}{|c|}{ Non-sepsis $(n=42)$} & \multicolumn{3}{|c|}{ Sepsis $(n=22)$} & \multicolumn{3}{|c|}{ Septic shock $(n=34)$} & \multirow{3}{*}{$\begin{array}{l}\text { p- } \\
\text { value } \\
< \\
0.001\end{array}$} \\
\hline & \multirow{2}{*}{$\begin{array}{l}\mathbf{n} \\
42\end{array}$} & \multicolumn{2}{|c|}{ median (IQR) } & \multirow{2}{*}{$\begin{array}{l}\mathbf{n} \\
22\end{array}$} & \multicolumn{2}{|c|}{ median (IQR) } & \multirow{2}{*}{$\begin{array}{l}\mathbf{n} \\
34\end{array}$} & \multicolumn{2}{|c|}{ median (IQR) } & \\
\hline & & 19.0 & $(10.9-35.8)$ & & 49.1 & $(34.4-89.7)$ & & 168.0 & $\begin{array}{l}(77.6- \\
295.0)\end{array}$ & \\
\hline $\mathrm{mAM}(\mathrm{pmol} / \mathrm{L})$ & 42 & 2.2 & $(1.2-3.7)$ & 22 & 4.7 & $(3.0-8.2)$ & 34 & 10.7 & $(6.4-23.1)$ & $\begin{array}{l}<.001 \\
0.00\end{array}$ \\
\hline $\begin{array}{l}\text { Procalcitonin } \\
(\propto \mathrm{g} / \mathrm{L})\end{array}$ & 37 & 0.3 & $(0.1-1.6)$ & 22 & 3.5 & $(0.5-13.4)$ & 34 & 23.2 & $(2.1-61.2)$ & $\begin{array}{l}<.001 \\
0.00\end{array}$ \\
\hline Presepsin (ng/L) & 36 & 663 & $\begin{array}{l}(298.5- \\
1231)\end{array}$ & 22 & 942 & $(446-3188)$ & 33 & 1443 & $\begin{array}{l}(662- \\
2447)\end{array}$ & 0.064 \\
\hline SOFA score & 42 & 6 & $(3-8)$ & 21 & 7 & $(6-11)$ & 34 & 11 & $(8-13)$ & $\dot{0} 0001$ \\
\hline $\begin{array}{l}\text { Lactate } \\
(\mathrm{mmol} / \mathrm{L})\end{array}$ & 42 & 1.3 & $(1.0-2.2)$ & 22 & 1.3 & $(1.0-1.9)$ & 34 & 3.7 & $(2.3-5.5)$ & $\begin{array}{l}<.001 \\
0\end{array}$ \\
\hline WBC $(/ \mathrm{mL})$ & 41 & $\begin{array}{l}10 \\
600\end{array}$ & $\begin{array}{l}(8125-14 \\
525)\end{array}$ & 21 & 9200 & $\begin{array}{l}(1950-17 \\
050)\end{array}$ & 34 & $\begin{array}{l}10 \\
400\end{array}$ & $\begin{array}{l}(4125-15 \\
950)\end{array}$ & 0.76 \\
\hline $\mathrm{CRP}(\mathrm{mg} / \mathrm{mL})$ & 39 & 3.9 & $(0.8-10.8)$ & 21 & 12.3 & $(7.7-20.7)$ & 34 & 18.6 & $(8.9-27.5)$ & $\begin{array}{l}<.001 \\
0.00\end{array}$ \\
\hline $\begin{array}{l}\text { Platelet ( } \\
\left.10^{9} / \mathrm{L}\right)\end{array}$ & 41 & 178 & $(111-203)$ & 21 & 120 & $(34-246)$ & 34 & 99 & $(34-145)$ & 0.002 \\
\hline PT-INR & 39 & 1.37 & $(1.11-1.84)$ & 20 & 1.28 & $(1.11-1.57)$ & 34 & 1.53 & $\begin{array}{l}(1.24- \\
1.87)\end{array}$ & 0.049 \\
\hline $\begin{array}{l}\text { Creatinine } \\
\text { (mg/dL) }\end{array}$ & 41 & 0.9 & $(0.7-2.2)$ & 21 & 1.3 & $(0.7-1.6)$ & 34 & 2.4 & $(1.1-2.9)$ & 0.006 \\
\hline $\begin{array}{l}\text { Data are express } \\
\text { interquartile rang } \\
\text { WBC, white bloo }\end{array}$ & 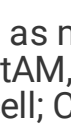 & $\begin{array}{l}\text { lian } \\
\text { tal a } \\
\text { C-re }\end{array}$ & $\begin{array}{l}\text { and analy } \\
\text { medullin; } \\
\text { e protein; } \mathrm{F}\end{array}$ & rith & $\begin{array}{l}\text { e Kru } \\
\text { lome } \\
\text { hrom }\end{array}$ & $\begin{array}{l}\text { I-Wallis test } \\
\text { in; SOFA, se } \\
\text { time-intern }\end{array}$ & it & $\begin{array}{l}\text { sive } \\
\text { rgan } \\
\text { mali }\end{array}$ & $\begin{array}{l}\text { unit; IQR; } \\
\text { ure assess } \\
\text { ratio. }\end{array}$ & \\
\hline
\end{tabular}

Clinical parameters including laboratory results, SOFA scores, and AM on ICU admission are presented in Table 2. tAM, mAM, PCT, PSEP, SOFA scores, and lactate levels, biomarkers of sepsis, are shown in Fig. 2. tAM, mAM, PCT, SOFA scores, and lactate levels increased significantly according to the severity of sepsis. tAM, mAM, and PCT levels were particularly divergent between patients categorized as non-sepsis and those categorized as sepsis/septic shock. There was no significant difference in SOFA score, PSEP, and lactate levels between the non-sepsis and sepsis groups (Fig. 2 and Table 2). Comparisons of tAM and mAM levels at various time points post-admission for short- and long-term observation patients classified as non-sepsis and sepsis/septic shock are shown in Fig. 3a and 3b. tAM and mAM in the septic/septic shock group were significantly higher than those in the non-sepsis group within 24 hours of admission and from admission to Day 3, respectively. 
ROC analysis for the diagnosis of sepsis and septic shock

Table 3

Diagnostic performance of each clinical parameter in sepsis/septic shock patients at ICU admission

\begin{tabular}{|c|c|c|c|c|c|c|c|c|c|c|}
\hline $\begin{array}{l}\text { Clinical } \\
\text { parameters }\end{array}$ & Cutoff & \multicolumn{2}{|c|}{ AUC (95\% Cl) } & \multirow{2}{*}{$\begin{array}{l}\text { Sensitivity } \\
\text { (\%) } \\
76.8\end{array}$} & \multirow{2}{*}{$\begin{array}{l}\text { Specificity } \\
\text { (\%) } \\
81.0\end{array}$} & \multirow{2}{*}{$\begin{array}{l}\text { PPV } \\
(\%)\end{array}$} & \multirow{2}{*}{$\begin{array}{l}\text { NPV } \\
(\%)\end{array}$} & \multirow{2}{*}{$\begin{array}{l}\text { LR+ } \\
4.03\end{array}$} & \multirow{2}{*}{$\begin{array}{l}\text { LR- } \\
0.29\end{array}$} & \multirow{2}{*}{$\begin{array}{l}\text { p- } \\
\text { value } \\
< \\
0.001\end{array}$} \\
\hline tAM & $39.6 \mathrm{pmol} / \mathrm{L}$ & 0.879 & $\begin{array}{l}(0.814- \\
0.945)\end{array}$ & & & & & & & \\
\hline $\mathrm{mAM}$ & $5.1 \mathrm{pmol} / \mathrm{L}$ & 0.848 & $\begin{array}{l}(0.773- \\
0.923)\end{array}$ & 69.6 & 85.7 & 86.7 & 67.9 & 4.87 & 0.35 & $\begin{array}{l}<.001 \\
0 .\end{array}$ \\
\hline Procalcitonin & $1.3 \propto \mathrm{g} / \mathrm{L}$ & 0.822 & $\begin{array}{l}(0.737- \\
0.907)\end{array}$ & 74.1 & 75.7 & 81.6 & 66.7 & 3.05 & 0.34 & $\begin{array}{l}<.001 \\
0.00\end{array}$ \\
\hline Presepsin & $1270 \mathrm{ng} / \mathrm{L}$ & 0.682 & $\begin{array}{l}(0.570- \\
0.794)\end{array}$ & 50.9 & 86.1 & 84.8 & 53.4 & 3.67 & 0.57 & 0.622 \\
\hline SOFA score & 8 & 0.772 & $\begin{array}{l}(0.679- \\
0.866)\end{array}$ & 60.0 & 83.3 & 82.5 & 61.4 & 3.60 & 0.48 & 0.370 \\
\hline Lactate & $1.8 \mathrm{mmol} / \mathrm{L}$ & 0.664 & $\begin{array}{l}(0.553- \\
0.775)\end{array}$ & 66.1 & 69.1 & 74.0 & 60.4 & 2.13 & 0.49 & $\begin{array}{l}<.001 \\
0.00\end{array}$ \\
\hline WBC & $8700 / \mathrm{mL}$ & 0.553 & $\begin{array}{l}(0.437- \\
0.669)\end{array}$ & 45.5 & 68.3 & 65.8 & 48.3 & 1.43 & 0.80 & 0.021 \\
\hline CRP & $7.4 \mathrm{mg} / \mathrm{dL}$ & 0.764 & $\begin{array}{l}(0.663- \\
0.865)\end{array}$ & 78.2 & 71.8 & 79.6 & 70.0 & 2.77 & 0.30 & $\begin{array}{l}<.001 \\
0.00\end{array}$ \\
\hline Platelet & $134 \times 10^{9} / \mathrm{L}$ & 0.693 & $\begin{array}{l}(0.586- \\
0.800)\end{array}$ & 67.3 & 70.7 & 75.5 & 61.7 & 2.30 & 0.46 & 0.004 \\
\hline PT-INR & 1.26 & 0.532 & $\begin{array}{l}(0.405- \\
0.659)\end{array}$ & 64.8 & 46.2 & 62.5 & 48.6 & 1.20 & 0.76 & $<.001$ \\
\hline Creatinine & $1.3 \mathrm{mg} / \mathrm{dL}$ & 0.638 & $\begin{array}{l}(0.521- \\
0.755)\end{array}$ & 58.2 & 68.3 & 71.1 & 54.9 & 1.83 & 0.61 & 0.002 \\
\hline $\begin{array}{l}\text { ICU, intensive } \\
\text { assessment; } V \\
\text { AUC, area und } \\
\text { positive likelih }\end{array}$ & $\begin{array}{l}\text { unit; tAM, } \\
\text {, white blo } \\
\text { urve; Cl, co } \\
\text { ratio; LR-, }\end{array}$ & $\begin{array}{l}\text { adre } \\
\text { all; CR } \\
\text { ince i }\end{array}$ & $\begin{array}{l}\text { edullin; } \\
\text { reactive } \\
\text { lal; PPV, } \\
\text { hood rat }\end{array}$ & $\begin{array}{l}\text { M, mature } \\
\text { tein; PT-IN } \\
\text { sitive pred }\end{array}$ & $\begin{array}{l}\text { enomedul } \\
\text { rothrombi } \\
\text { e value; N }\end{array}$ & $\begin{array}{l}\text { so } \\
\text { ime- } \\
\text { neg }\end{array}$ & $\begin{array}{l}\text { sequ } \\
\text { rnat } \\
\text { e pr }\end{array}$ & $\begin{array}{l}\text { tial o } \\
\text { al no } \\
\text { ctive }\end{array}$ & $\begin{array}{l}\text { In fa } \\
\text { alize } \\
\text { lue; L }\end{array}$ & atio; \\
\hline
\end{tabular}


Table 4

Significance level of pairwise comparisons of ROC curves in sepsis/septic shock patients at ICU admission

\begin{tabular}{|c|c|c|c|c|c|c|c|}
\hline \multirow[t]{2}{*}{ Clinical parameters } & \multirow[t]{2}{*}{ AUC } & \multicolumn{6}{|c|}{ p-value of pairwise comparison of ROC curves } \\
\hline & & tAM & mAM & SOFA score & Lactate & Procalcitonin & Presepsin \\
\hline tAM & 0.872 & - & 0.093 & 0.010 & $<0.001$ & 0.221 & $<0.001$ \\
\hline mAM & 0.824 & - & - & 0.110 & 0.008 & 0.889 & 0.009 \\
\hline SOFA score & 0.734 & - & - & - & 0.325 & 0.107 & 0.283 \\
\hline Lactate & 0.656 & - & - & - & - & 0.024 & 0.867 \\
\hline Procalcitonin & 0.817 & - & - & - & - & - & 0.014 \\
\hline Presepsin & 0.669 & - & - & - & - & - & - \\
\hline
\end{tabular}


Table 5

Diagnostic performance of each clinical parameter in septic shock patients at ICU admission

\begin{tabular}{|c|c|c|c|c|c|c|c|c|c|c|}
\hline $\begin{array}{l}\text { Clinical } \\
\text { parameters }\end{array}$ & Cutoff & \multicolumn{2}{|c|}{ AUC (95\% Cl) } & \multirow{2}{*}{$\begin{array}{l}\text { Sensitivity } \\
\text { (\%) } \\
82.9\end{array}$} & \multirow{2}{*}{$\begin{array}{l}\text { Specificity } \\
\text { (\%) } \\
82.5\end{array}$} & \multirow{2}{*}{$\begin{array}{l}\text { PPV } \\
\text { (\%) } \\
72.5\end{array}$} & \multirow{2}{*}{$\begin{array}{l}\text { NPV } \\
\text { (\%) } \\
89.7\end{array}$} & \multirow{2}{*}{$\begin{array}{l}\text { LR+ } \\
\\
4.75\end{array}$} & \multirow{2}{*}{$\begin{array}{l}\text { LR- } \\
0.21\end{array}$} & \multirow{2}{*}{$\begin{array}{l}\text { p- } \\
\text { value } \\
< \\
0.001\end{array}$} \\
\hline tAM & $57.9 \mathrm{pmol} / \mathrm{L}$ & 0.858 & $\begin{array}{l}(0.775- \\
0.941)\end{array}$ & & & & & & & \\
\hline mAM & $6.1 \mathrm{pmol} / \mathrm{L}$ & 0.830 & $\begin{array}{l}(0.745- \\
0.915)\end{array}$ & 77.1 & 81.0 & 69.2 & 86.4 & 4.05 & 0.28 & $<.001$ \\
\hline Procalcitonin & $9.6 \propto \mathrm{g} / \mathrm{L}$ & 0.811 & $\begin{array}{l}(0.722- \\
0.900)\end{array}$ & 66.7 & 86.2 & 73.3 & 82.0 & 4.83 & 0.39 & $<.001$ \\
\hline Presepsin & $1270 \mathrm{ng} / \mathrm{L}$ & 0.661 & $\begin{array}{l}(0.547- \\
0.775)\end{array}$ & 55.9 & 75.4 & 57.6 & 74.1 & 2.28 & 0.58 & 0.020 \\
\hline SOFA score & 8 & 0.816 & $\begin{array}{l}(0.730- \\
0.902)\end{array}$ & 74.3 & 77.4 & 65.0 & 84.2 & 3.29 & 0.33 & 0.657 \\
\hline Lactate & $1.9 \mathrm{mmol} / \mathrm{L}$ & 0.828 & $\begin{array}{l}(0.740- \\
0.916)\end{array}$ & 88.6 & 74.6 & 66.0 & 92.2 & 3.49 & 0.15 & $<.001$ \\
\hline WBC & $6600 / \mathrm{mL}$ & 0.529 & $\begin{array}{l}(0.401- \\
0.657)\end{array}$ & 37.1 & 82.0 & 54.2 & 69.4 & 2.06 & 0.77 & $<.001$ \\
\hline CRP & $14.8 \mathrm{mg} / \mathrm{dL}$ & 0.734 & $\begin{array}{l}(0.630- \\
0.838)\end{array}$ & 65.7 & 76.3 & 62.2 & 78.9 & 2.77 & 0.45 & $<.001$ \\
\hline Platelet & $134 \times 10^{9} / \mathrm{L}$ & 0.701 & $\begin{array}{l}(0.596- \\
0.805)\end{array}$ & 74.3 & 62.3 & 53.1 & 80.9 & 1.97 & 0.41 & $<.001$ \\
\hline PT-INR & 1.35 & 0.633 & $\begin{array}{l}(0.521- \\
0.746)\end{array}$ & 68.6 & 53.5 & 47.1 & 73.8 & 1.47 & 0.59 & $<.001$ \\
\hline Creatinine & $1.8 \mathrm{mg} / \mathrm{dL}$ & 0.698 & $\begin{array}{l}(0.591- \\
0.804)\end{array}$ & 62.9 & 75.4 & 59.5 & 78.0 & 2.56 & 0.49 & 0.006 \\
\hline $\begin{array}{l}\text { ICU, intensive } \\
\text { assessment; } \\
\text { AUC, area ung } \\
\text { positive likeli }\end{array}$ & $\begin{array}{l}\text { e unit; tAM, } \\
\text { c, white bloc } \\
\text { curve; Cl, Co } \\
\text { d ratio; LR-, }\end{array}$ & $\begin{array}{l}\text { I adre } \\
\text { ell; CP } \\
\text { ence } \\
\text { ative }\end{array}$ & $\begin{array}{l}\text { edullin; } \\
\text { reactive } \\
\text { val; PPI } \\
\text { nood ra }\end{array}$ & $\begin{array}{l}\text { M, matur } \\
\text { tein; PT-II } \\
\text { sitive pre }\end{array}$ & $\begin{array}{l}\text { renomedu } \\
\text { rothromb } \\
\text { /e value; } ~\end{array}$ & $\begin{array}{l}\text { SOF } \\
\text { ime-i } \\
\text { ineg }\end{array}$ & $\begin{array}{l}\text { sequ } \\
\text { rnati } \\
\text { le pr }\end{array}$ & $\begin{array}{l}\text { tial o } \\
\text { al no } \\
\text { ctive }\end{array}$ & $\begin{array}{l}\text { In fa } \\
\text { alize } \\
\text { lue; }\end{array}$ & tio; \\
\hline
\end{tabular}


Table 6

Significance level of pairwise comparisons of ROC curves in septic shock patients at ICU admission

\begin{tabular}{|c|c|c|c|c|c|c|c|}
\hline \multirow[t]{2}{*}{ Clinical parameters } & \multirow[t]{2}{*}{ AUC } & \multicolumn{6}{|c|}{ p-value of pairwise comparison of ROC curves } \\
\hline & & tAM & mAM & SOFA score & Lactate & Procalcitonin & Presepsin \\
\hline tAM & 0.859 & - & 0.144 & 0.276 & 0.575 & 0.312 & 0.003 \\
\hline mAM & 0.815 & - & - & 0.730 & 0.836 & 0.955 & 0.018 \\
\hline SOFA score & 0.793 & - & - & - & 0.626 & 0.767 & 0.033 \\
\hline Lactate & 0.828 & - & - & - & - & 0.815 & 0.012 \\
\hline Procalcitonin & 0.813 & - & - & - & - & - & 0.025 \\
\hline Presepsin & 0.657 & - & - & - & - & - & - \\
\hline
\end{tabular}

To compare the diagnostic accuracy of sepsis and septic shock using each biomarker, we performed an ROC curve analysis and determined the AUCs. The AUCs of tAM, mAM, PCT, PSEP, SOFA scores, and lactate levels for the diagnosis of sepsis/septic shock were $0.879,0.848,0.822,0.682,0.772$, and 0.664 , respectively (Fig. $4 a$ and Table 3 ). The AUCs of tAM and mAM for the diagnosis of sepsis were significantly higher than those of lactate $(p<0.001$ and $p=0.008$, respectively) and PSEP ( $<0.001$ and $p=0.009$, respectively) (Table 4). The cutoff points of tAM and mAM for the diagnosis of sepsis were $39.6 \mathrm{pmol} / \mathrm{L}(\approx 237.6 \mathrm{ng} / \mathrm{L}$ ) (AUC: $0.879,95 \% \mathrm{Cl}: 0.773-0.923$, sensitivity: $76.8 \%$, specificity: $81.0 \%$ ) and $5.1 \mathrm{pmol} / \mathrm{L}(\approx 30.6 \mathrm{ng} / \mathrm{L})$ (AUC: $0.848,95 \% \mathrm{Cl}: 0.814--0.945$, sensitivity: $69.6 \%$, specificity: $85.7 \%$ ), respectively (Table 3). The positive likelihood ratio (LR+) of mAM for the diagnosis of sepsis was 4.87 , which was the highest value among all clinical parameters (Table 3). The negative likelihood ratio (LR-) of tAM for the diagnosis of sepsis was 0.29 , which was the lowest value among all clinical parameters (Table 3 ).

The AUCs of tAM, mAM, PCT, PSEP, SOFA score, and lactate levels for the diagnosis of septic shock were $0.858,0.830$, $0.811,0.661,0.816$, and 0.828 , respectively. (Fig. $4 \mathrm{~b}$ and Table 5). The AUCs of tAM and mAM were significantly higher than those of PSEP ( $p=0.0033$ and $p=0.0184$, respectively) (Table 6). The cutoff points of tAM and mAM for the diagnosis of septic shock were $57.9 \mathrm{pmol} / \mathrm{L}(\approx 347.4 \mathrm{ng} / \mathrm{L})$ and $6.1 \mathrm{pmol} / \mathrm{L}(\approx 36.6 \mathrm{ng} / \mathrm{L})$, respectively. The highest LR+ for the diagnosis of septic shock was 4.83 in PCT.

\section{ROC analysis to predict 28-day mortality associated with sepsis and septic shock}


Table 7

Comparison of clinical parameters of survivors and non-survivors in sepsis/septic shock (Day 1)

\begin{tabular}{|c|c|c|c|c|c|c|c|c|}
\hline \multirow{3}{*}{$\begin{array}{l}\text { Clinical parameters } \\
\text { tAM (pmol/L) }\end{array}$} & \multicolumn{3}{|c|}{ Survivor } & \multicolumn{3}{|c|}{ Non-survivor } & \multirow{3}{*}{$\begin{array}{l}\text { p-value } \\
0.180\end{array}$} & \multirow[t]{3}{*}{ Power } \\
\hline & \multirow{2}{*}{$\mathbf{n}$} & \multicolumn{2}{|c|}{ median (IQR) } & \multirow{2}{*}{8} & \multicolumn{2}{|c|}{ median (IQR) } & & \\
\hline & & 57.9 & $(34.9-178.0)$ & & 153 & $(87.3-304.0)$ & & \\
\hline mAM (pmol/L) & 17 & 6.4 & $(3.0-14.3)$ & 8 & 5.9 & $(3.9-7.4)$ & 0.560 & \\
\hline Procalcitonin ( $\propto \mathrm{g} / \mathrm{L})$ & 17 & 10.4 & $(1.2-31.0)$ & 8 & 16.8 & $(1.0-93.6)$ & 0.462 & \\
\hline Presepsin (ng/L) & 17 & 664 & $(377-1400)$ & 8 & 960 & $(571-2027)$ & 0.396 & \\
\hline SOFA score & 17 & 7 & $(5.8-11.3)$ & 8 & 11.5 & $(11.0-13.5)$ & 0.113 & \\
\hline Lactate (mmol/L) & 17 & 1.9 & $(0.9-2.8)$ & 8 & 4.0 & $(3.3-5.8)$ & 0.010 & 0.579 \\
\hline WBC (/mL) & 17 & 10400 & $(3150-16475)$ & 8 & 12250 & $(4900-23$ 150) & 0.522 & \\
\hline CRP (mg/mL) & 17 & 13.6 & $(5.1-23.7)$ & 8 & 15.4 & $(8.7-19.2)$ & 0.600 & \\
\hline Platelet $\left(\times 10^{9} / \mathrm{L}\right)$ & 17 & 109 & $(38-187)$ & 8 & 138 & $(76-198)$ & 0.705 & \\
\hline PT-INR & 17 & 1.30 & $(1.16-1.70)$ & 8 & 1.61 & $(1.33-2.43)$ & 0.081 & \\
\hline Creatinine (mg/dL) & 17 & 1.2 & $(1.0-1.6)$ & 8 & 2.1 & $(1.2-2.8)$ & 0.200 & \\
\hline \multicolumn{9}{|c|}{$\begin{array}{l}\text { Data are expressed as the median (IQR) and analyzed with a Mann-Whitney U-test. Retrospective power analyses } \\
\text { were performed with G*Power Post hoc t-tests (TwO-tails, a = 0.05). IQR; interquartile range; tAM, total adrenomedullin; } \\
\text { mAM, mature adrenomedullin; SOFA, sequential organ failure assessment; WBC, white blood cell; CRP, C-reactive } \\
\text { protein; PT-INR, prothrombin time-international normalized ratio. }\end{array}$} \\
\hline
\end{tabular}


Table 8

Comparison of clinical parameters of survivors and non-survivors in sepsis/septic shock (Day 3)

\begin{tabular}{|c|c|c|c|c|c|c|c|c|}
\hline \multirow{3}{*}{$\begin{array}{l}\text { Clinical parameters } \\
\text { tAM (pmol/L) }\end{array}$} & \multicolumn{3}{|c|}{ Survivor } & \multicolumn{3}{|c|}{ Non-survivor } & \multirow{3}{*}{$\begin{array}{l}\text { p-value } \\
0.002\end{array}$} & \multirow{3}{*}{$\begin{array}{l}\text { Power } \\
0.960\end{array}$} \\
\hline & \multirow{2}{*}{ n } & \multicolumn{2}{|c|}{ median (IQR) } & \multirow{2}{*}{ n } & \multicolumn{2}{|c|}{ median (IQR) } & & \\
\hline & & 22.0 & $(18.1-40.3)$ & & 132 & $(102-221)$ & & \\
\hline mAM (pmol/L) & 17 & 3.2 & $(2.2-4.9)$ & 6 & 10.8 & $(8.7-14.2)$ & 0.005 & 0.924 \\
\hline Procalcitonin ( $\propto \mathrm{g} / \mathrm{L})$ & 14 & 12.6 & $(3.7-19.7)$ & 6 & 40.7 & $(7.7-127.0)$ & 0.288 & \\
\hline Presepsin (ng/L) & 12 & 632 & $(285-884)$ & 6 & 1050 & $(746-2013)$ & 0.090 & \\
\hline SOFA score & 17 & 5 & $(4-9.3)$ & 6 & 12.5 & $(12-15)$ & 0.003 & 0.988 \\
\hline Lactate (mmol/L) & 17 & 1.2 & $(1.1-1.5)$ & 6 & 2.1 & $(1.8-2.6)$ & 0.036 & 0.615 \\
\hline WBC (/mL) & 17 & 10900 & $(6400-21$ 100) & 6 & 9550 & $(5000-24600)$ & 0.529 & \\
\hline CRP (mg/mL) & 17 & 12.3 & $(6.4-22.3)$ & 6 & 19.5 & $(9.5-34.5)$ & 0.363 & \\
\hline Platelet $\left(\times 10^{9} / \mathrm{L}\right)$ & 17 & 69 & $(42-200)$ & 6 & 63 & $(46-102)$ & 0.575 & \\
\hline PT-INR & 15 & 1.24 & $(1.13-1.35)$ & 6 & 1.41 & $(1.24-1.52)$ & 0.094 & \\
\hline Creatinine (mg/dL) & 17 & 1.0 & $(0.5-1.7)$ & 6 & 1.2 & $(0.8-2.7)$ & 0.345 & \\
\hline \multicolumn{9}{|c|}{$\begin{array}{l}\text { Data are expressed as the median (IQR) and analyzeded with a Mann-Whitney U-test. Retrospective power analyses } \\
\text { were performed with G*Power Post hoc t-tests (TwO-tails, a = 0.05). IQR; interquartile range; tAM, total adrenomedullin; } \\
\text { mAM, mature adrenomedullin; SOFA, sequential organ failure assessment; WBC, white blood cell; CRP, C-reactive } \\
\text { protein; PT-INR, prothrombin time-international normalized ratio. }\end{array}$} \\
\hline
\end{tabular}

In sepsis/septic shock patients, tAM and mAM levels in non-survivors on Day 3 were significantly higher than those in survivors, whereas there was no significant difference in tAM and mAM levels on admission day (Day 1) between survivors and non-survivors (Tables 7 and 8 ).

Table 9

Prognostic performance of each clinical biomarker for 28-day mortality for patients with sepsis/septic shock

\begin{tabular}{|c|c|c|c|}
\hline \multirow[t]{2}{*}{ Clinical parameters } & \multicolumn{3}{|l|}{ AUC (p-value) } \\
\hline & Day $1(n=25)$ & Day $3(n=23)$ & Day $5(n=11)$ \\
\hline tAM & $0.669(0.173)$ & $0.931(<0.001)$ & $0.911(<0.001)$ \\
\hline mAM & $0.574(0.553)$ & $0.892(<0.001)$ & $0.667(0.011)$ \\
\hline SOFA score & $0.669(0.108)$ & $0.922(<0.001)$ & $0.889(<0.001)$ \\
\hline Lactate & $0.824(<0.001)$ & $0.794(0.013)$ & $0.889(<0.001)$ \\
\hline Platelet & $0.548(0.721)$ & $0.578(0.554)$ & $0.556(<0.001)$ \\
\hline \multicolumn{4}{|c|}{$\begin{array}{l}\text { AUC, area under curve. tAM, total adrenomedullin; mAM, mature adrenomedullin; SOFA, } \\
\text { sequential organ failure assessment. }\end{array}$} \\
\hline
\end{tabular}


Table 10

Prognostic performance of AM for 28-day mortality for patients with sepsis/septic shock

\begin{tabular}{|c|c|c|c|c|c|c|c|c|}
\hline Biomarkers & Day & Cutoff (pmol/L) & AUC (95\% Cl) & Sensitivity (\%) & Specificity (\%) & PPV (\%) & NPV (\%) & \begin{tabular}{l|l} 
LR+ & LR-
\end{tabular} \\
\hline \multirow[t]{3}{*}{ tAM } & 1 & 64.2 & $0.669 \mid(0.426-0.912)$ & 87.5 & 58.8 & 50.0 & 90.9 & \begin{tabular}{l|l|l|}
2.12 & 0.21
\end{tabular} \\
\hline & 3 & 72.5 & $0.931(0.791-1.000)$ & 83.3 & 100 & 100 & 94.4 & 0.17 \\
\hline & 5 & 27.5 & $0.911(0.758-1.000)$ & 100 & 80.0 & 50.0 & 100 & 5.000 \\
\hline \multirow[t]{3}{*}{ mAM } & 1 & 6.5 & $0.574(0.331-0.816)$ & 75.0 & 47.1 & 40.0 & 80.0 & 1.420. \\
\hline & 3 & 5.4 & $0.892(0.714-1.000)$ & 83.3 & 88.2 & 71.0 & 94.0 & \begin{tabular}{ll|l|l|}
7.08 & 0.19
\end{tabular} \\
\hline & 5 & 5.5 & $0.844(0.579-1.000)$ & 66.7 & 93.3 & 66.7 & 93.3 & 9.960 .36 \\
\hline
\end{tabular}

AM, adrenomedullin; AUC, area under curve; $\mathrm{Cl}$, Confidence interval; tAM, total adrenomedullin; mAM, mature adrenomedullin; PPV, positive predictive value; NPV, negative predictive value; LR+, positive likelihood ratio; LR-, negative likelihood ratio.

ROC analysis was performed to predict 28-day mortality in septic patients (Table 9). The AUCs of tAM, mAM, and SOFA scores on Day 3 were $0.931,0.892$, and 0.922 , respectively, whereas lactate was the only potential for biomarker (AUC of 0.824 ) to predict 28-day mortality on Day 1 . The cutoff points of tAM and mAM for 28-day mortality on Day 3 were $72.5 \mathrm{pmol} / \mathrm{L}(\approx 435.0 \mathrm{ng} / \mathrm{L})$ (sensitivity: $83.3 \%$, specificity: $100 \%$ ) and $5.4 \mathrm{pmol} / \mathrm{L}(\approx 32.4 \mathrm{ng} / \mathrm{L})$ (sensitivity: $83.3 \%$, specificity: $88.2 \%)$, respectively (Table 10$)$.

\section{Discussion}

This is a prospective, single-center study investigating the diagnostic performance of plasma adrenomedullin (total and mature AM) for sepsis and septic shock defined by Sepsis-3, and the prognostic performance of AM for 28-day mortality of patients with sepsis. In this study, we demonstrated that (1) AM is a competent biomarker with the greatest diagnostic accuracy for sepsis and septic shock among the biomarkers we evaluated, (2) AM can accurately diagnose sepsis and septic shock at ICU admission, and (3) AM has the potential to prognose 28-day mortality for patients with sepsis, especially from Day 2 to Day 5 post-ICU admission, as do SOFA score and lactate levels.

\section{Sepsis-3 definitions and sepsis biomarkers}

Sepsis is a life-threatening event caused by microbial infection-induced multiple organ failure. In 1992, the American College of Chest Physicians and the Society of Critical Care Medicine (SCCM) first proposed the definition and diagnosis of sepsis based on the SIRS criteria, referred to as Sepsis-1 [35]. Subsequently, in 2001, the SCCM, the American College of Chest Physicians, the European Society of Intensive Care Medicine, the American Thoracic Society, and the Surgical Infection Society refined the diagnostic criteria of sepsis, called Sepsis-2, and defined "severe sepsis" [36]. The SCCM and the European Society of Intensive Care Medicine published the Sepsis-3 definition and diagnostic criteria in 2016, which removed SIRS and severe sepsis from the original Sepsis-1 concept, and redefined sepsis using the SOFA score [29]. As described by Fang and colleagues, when two or more SIRS criteria (Sepsis-1 and - 2) or the increment in SOFA score $\geq 2$ (Sepsis-3) were applied to predict 21-day all-cause mortality in infected patients without prior chronic organ dysfunction, the sensitivity was $96.0 \%$ or $91.0 \%$, and the specificity was $8.3 \%$ or $21.9 \%$, respectively [37]. Sepsis-3 diagnostic criteria may narrow the sepsis population, because the SOFA score represents the severity of the condition, whereas the SIRS score represents a clinically evident host response to infection.

Diagnosis by Sepsis-3 definitions is based on identifying the infection and SOFA scoring. Although hematological, biochemical, and microbiological laboratory tests are essential to diagnose sepsis, culture-based infection diagnosis takes time [8], and failure to identify sepsis in the early stages delays effective treatment, resulting in high mortality. Thus, major efforts have been made to find biomarkers that allow early diagnosis of sepsis $[7,8]$. Recently, TREM-1, HMGB1, PCT, and PSEP were identified and validated as sepsis biomarkers. PCT, widely regard as one of the most useful sepsis biomarkers, is recommended by the Surviving Sepsis Campaign Guideline (SSCG) [29]; however, PCT is not an ideal diagnostic biomarker. For patients on steroids, or patients with local infection alone or infection with atypical bacteria, PCT results can be falsely negative. Moreover, PCT levels may increase after cardiac arrest or surgery, and in 
patients with severe trauma [8]. Thus, in this study, we evaluated the blood concentration of AM, which is elevated in sepsis [19-28], as an additional potential biomarker to diagnose sepsis and septic shock defined by Sepsis-3.

\section{Two molecular forms of AM in circulation}

Kitamura et al. reported that two major molecular forms of AM circulate in the blood of humans, biologically active mature AM (mAM) with an amidated C-terminus, and inactive intermediate AM (iAM) with a non-amidated C-terminal glycine $[30,31]$. Numerous prior studies assessed AM levels in plasma and tissue, but the immunoreactive AM detected in these studies was the tAM level, which includes both mAM and IAM. The clinical significance of iAM is still unclear. Some reports showed similar changes in $\operatorname{mAM}$ and IAM $[33,38,39]$. Although iAM is biologically inactive, one study demonstrated that following conversion to $\mathrm{mAM}$, it exerted vasodilatory action in isolated rat aortas ex vivo and took much longer to achieve maximum relaxation than mAM, suggesting that iAM may act as a hormone reservoir [40]. Bioactive AM would be an informative biomarker; however, its short half-life (approximately 22 minutes) and the AMbinding protein, complement factor $\mathrm{H}$, hinder the reliable measurement of bioactive $\mathrm{AM}[27,28,41]$. To address these problems, mid-regional pro-adrenomedullin (MR-proADM or proADM), another fragment of the AM precursor peptide, has been introduced as an alternative biomarker $[27,28]$. Although it had been presumed that MR-proADM represented the sum of synthesized iAM and mAM, the ratio between MR-proADM and bioactive AM can vary, thus MR-proADM does not always reflect the actual amount of bioactive AM [40]. Recently, Weber et al. reported a new assay to reliably measure the bioactive mature form of AM (which they referred to as bio-ADM) [42], and bio-ADM in patients with sepsis is more strongly associated with clinical outcome relative to MR-proADM concentrations [24]. In our mAM assay, the limits of detection and quantitation were 0.133 and $0.085 \mathrm{pM}$, respectively, according to Clinical and Laboratory Standards Institute (CLSI) protocols. Intra- and inter-assay coefficients of variation were $1.8 \%$ and $5.1 \%$, respectively [33]. However, we did not assess the impact of complement factor $\mathrm{H}$ on our mAM assay. Thus, we believe our mAM assay is as reliable as the bio-ADM assay reported by Weber et al., unless complement factor $\mathrm{H}$ concentration is especially high.

\section{AM and other clinical study parameters}

Consistent with previous studies, we observed that at ICU admission, tAM and mAM were moderately elevated in patients with sepsis (without shock), whereas, they were highly elevated in patients with septic shock [19-28]. In contrast, SOFA score, PSEP, and lactate levels in patients with sepsis (without shock) were not significantly different from those in the non-sepsis group. The SOFA score and lactate levels of non-sepsis patients were elevated because the non-sepsis group contained patients who had severe diseases with organ failure or organ dysfunction such as acute heart failure, acute pancreatitis, acute hepatic failure, and acute interstitial pneumonitis. When non-sepsis patients were admitted to the ICU, the average PSEP level was $663 \mathrm{ng} / \mathrm{L}$, which was as same as cutoff value (670 ng/L) for sepsis diagnosis reported by Nakamura et al [43]. As described in previous reports [43, 44], this high PSEP level may be associated with renal dysfunction, because the creatinine level of non-sepsis patients was also elevated.

\section{Diagnostic performance of AM at ICU admission for patients with sepsis and septic shock, as defined by Sepsis-3}

Although multiple reports have claimed that bioactive AM and MR-proADM could prognose the severity of sepsis, the progression to septic shock, or sepsis-related mortality [19-28], there has been little mention of the performance of AM for diagnosing sepsis using the latest Sepsis-3 definitions. In this study, we found that among all clinical parameters examined, tAM had the highest diagnostic accuracy for sepsis at ICU admission, and the diagnostic accuracy of mAM for sepsis was also high (Table 3 ).

Bernal-Morell et al. reported that to diagnose sepsis, the AUCs of MR-proADM, CRP, PCT, and lactate were 0.771 (95\% Cl: 0.692-0.850), 0.643 (95\% Cl: 0.547-0.739), 0.695 (95\% Cl: 0.604-0.786), and 0.483 (95\% Cl: 0.383-0.583), respectively 
[45]. Wacker et al. reported that the AUC of PCT for sepsis diagnosis was 0.85 ( $95 \%$ Cl: $0.81-0.88$, sensitivity: $77 \%$, specificity: 79\%) [46]. Kondo et al. reported that the AUC of PCT and PSEP were 0.84 (95\% Cl: $0.81-0.87$, sensitivity: $80 \%$, specificity: $75 \%)$ and 0.87 (95\% Cl: 0.84-0.90, sensitivity: 84\%, specificity: 73\%), respectively [47]. These reports suggest that PCT and PSEP are high-quality, diagnostic biomarkers of sepsis. The diagnostic performance of PCT in our study was consistent with these previous reports. Additionally, we found that the ability of tAM and mAM to diagnose sepsis was equivalent to that of PCT; however, the diagnostic performance of PSEP was lower than that reported by previous studies. As described previously, the PSEP levels in the non-sepsis group in our study may have been elevated due to renal dysfunction [44].

The diagnostic performances of tAM and mAM for septic shock were highest among the clinical parameters evaluated at ICU admission, and were equivalent to the diagnostic performance of lactate and PCT for septic shock. Chen et al. reported that the AUC for the diagnostic performance of AM for severe sepsis and septic shock defined by Sepsis-2 was 0.847 (95\% Cl: 0.797-0.898, cutoff value: $41.24 \mathrm{ng} / \mathrm{L}$, sensitivity: 67.6\%, and specificity: $90.0 \%$ ) [25]. The AUC for diagnostic performance of AM for sepsis and septic shock in our study is consistent with previous reports, although we used Sepsis-3 diagnostic criteria and prior studies used Sepsis-1 or Sepsis-2 criteria. These findings suggest that AM levels increase as the severity of sepsis increases, thus, AM may be a suitable diagnostic biomarker for point-of-care testing.

\section{Predictive performance of AM for the prognosis of patients with sepsis}

Prognosing the clinical outcome of sepsis patients with MR-proADM and bioactive AM levels has also been described $[19-21,23,24,26,27]$. Chen et al. reported that the AUC of AM to predict the risk of in-hospital mortality was $0.773(95 \%$ Cl: $0.738-0.808$, cutoff value: $34.49 \mathrm{ng} / \mathrm{L}$, sensitivity: $81.6 \%$, specificity: $60.8 \%$ ) [26]. Marino et al. demonstrated that bioADM levels at ICU admission strongly correlated with 28-day mortality. Patients with ADM levels $>70$ ng/L had a 28-day survival rate of $55 \%$. For patients in this group whose ADM levels remained above $70 \mathrm{ng} / \mathrm{L}$ through Day 4 postadmission, the survival rate was $36 \%$. In contrast, when ADM levels were below $70 \mathrm{pg} / \mathrm{mL}$ the survival rate was $100 \%$ [24]. Furthermore, Mebazaa et al. reported that in patients with bio-ADM $>70 \mathrm{ng} / \mathrm{L}$ on admission, a decrease in bio-ADM levels to below $70 \mathrm{ng} / \mathrm{L}$ on Day 2 was associated with recovery of organ function on Day 7 and better 28-day outcomes (9.5\% mortality), whereas persistently elevated bio-ADM levels on Day 2 were associated with prolonged organ dysfunction and high 28-day mortality (38.1\% mortality) [20]. In our study, tAM and mAM levels on Day 1 could not predict 28-day sepsis-related mortality; however, their levels on Day 3 showed a high predictive value for sepsis-related mortality by ROC analysis. In fact, the AUCs of tAM and mAM were the highest among the biomarkers evaluated. These results suggest that both tAM and mAM levels reflect the severity of organ damage and mortality will increase if organ damage continues.

Previous reports suggested that increasing AM levels correlated worsening prognoses for patients with sepsis [48]. In contrast, AM can have a tissue protective effect in vitro and in vivo. Moreover, AM is highly anticipated as a new therapeutic agent for inflammatory bowel disease [10]. Although some preclinical animal studies have evaluated the effects of AM or AM antibody therapy for sepsis [49], it is still unclear if AM will have beneficial therapeutic effects for patients with sepsis. There is an ongoing, multi-center study evaluating AM-binding antibody therapy for sepsis (AdrenOSS-2) [50]. The results of this study may finally characterize the efficacy of AM therapy for patients with sepsis.

\section{Limitations}

Our study has several limitations. The effect of complement factor $\mathrm{H}$ on the mAM assay used in our study is not yet known. The sample size was small. The laboratory data, which are obtained in daily routine and used by the practicing clinicians (except for PSEP) were not measured using the same blood sample used for AM measurement. Furthermore, 
this study was performed as a small, single-center study focusing on the diagnostic performance of AM for sepsis and septic shock based on Sepsis-3 definitions. Thus, future randomized, controlled, multi-center studies should seek to verify our study results.

\section{Conclusions}

We found that AM plasma levels at ICU admission can diagnose sepsis and septic shock according to the latest Sepsis-3 definitions with accuracy that is high and comparable to PCT. Furthermore, except for ICU admission day, AM levels acquired during the first week of an ICU stay can also predict 28-day, sepsis-related mortality with accuracy comparable to predictions derived by co-assessing SOFA scores and lactate levels.

\section{Abbreviations}

AM: Adrenomedullin; ICU: Intensive care unit; ROC: Receiver operating characteristic; AUC: Area under the curve; PCT: Procalcitonin; PSEP: Presepsin; SOFA: Sequential organ failure assessment; CRP: C reactive protein; MAP: Mean atrial pressure; SIRS: Systemic inflammatory response syndrome; MR-proADM: Mid-region of adrenomedullin; APACHE II: Acute physiology and chronic health evaluation II; WBC: White blood cell; PT-INR: Prothrombin time-international normalized ratio; TREM-1: Triggering receptor expressed on myeloid cells 1; HMGB1: High mobility group box 1.

\section{Declarations}

\section{Ethics approval and consent to participate}

This study was conducted in accordance with the principles of the Declaration of Helsinki and was approved by the hospital ethical committee for human studies (Ref: 0-0317) on 1 December 2014 and retrospectively registered with Japanese Clinical Trial Registry "UMIN-CTR" (Ref: UMIN000036474). The general informed consent was obtained from all short-term observational patients and the written informed consent was obtained from all long-term observational patients.

\section{Consent for publication}

Not applicable.

\section{Availability of data and materials}

The datasets used and/or analyzed during the current study are available from the corresponding author on reasonable request.

\section{Competing Interests}

$\mathrm{KI}$ is an employee of Tosoh Corporation. The other authors declare no competing interests.

\section{Funding}

This study was supported by a Grant for Clinical Research from the University of Miyazaki Hospital.

\section{Authors' contributions}

TeY, TaY, and MT recruited patients and obtained clinical and laboratory samples. TeY and TM evaluated the data and drafted the manuscript. TeY, TM, and KI analyzed the data and performed the statistical analyses. TeY, TM, KK, and IT 
conceived the study and participated in study design and coordination. All authors revised, read, and approved the final manuscript.

\section{Acknowledgments}

This study is attributed to the Department of Anesthesiology, Intensive Care Unit and Internal Medicine, Faculty of Medicine, University of Miyazaki. The authors would like to thank Mariko Tokashiki, Noriko Hidaka, and Toshiko Watanabe for their technical advice and assistance in data collection in this study. We would like to thank Editage (www.editage.com) for English language editing.

\section{References}

1. Vincent JL, Jones G, David S, Olariu E, Cadwell KK. Frequency and mortality of septic shock in Europe and North America: a systematic review and meta-analysis. Crit Care. 2019;23:196.

2. Shankar-Hari M, Phillips GS, Levy ML, Seymour CW, Liu VX, Deutschman CS, et al. Sepsis Definitions Task Force. Developing a new definition and assessing new clinical criteria for septic shock: for the Third International Consensus Definitions for Sepsis and Septic Shock (Sepsis-3). JAMA. 2016;315:775-

3. Hotchkiss RS, Moldawer LL, Opal SM, Reinhart K, Turnbull IR, Vincent JL. Sepsis and septic shock. Nat Rev Dis Primers. 2016;2:16045.

4. Rhee C, Dantes R, Epstein L, Murphy DJ, Seymour CW, Iwashyna TJ, et al. Incidence and trends of sepsis in US hospitals using clinical vs claims data, 2009-2014. JAMA. 2017;318:1241-

5. Nguyen HB, Jaehne AK, Jayaprakash N, Semler MW, Hegab S, Yataco AC, et al. Early goal-directed therapy in severe sepsis and septic shock: insights and comparisons to ProCESS, ProMISe, and ARISE. Crit Care. 2016;20:160.

6. Chen CC, Chong CF, Liu YL, Chen KC, Wang TL. Risk stratification of severe sepsis patients in the emergency department. Emerg Med J. 2006;23:281-

7. Raveendran AV, Kumar A, Gangadharan S. Biomarkers and newer laboratory investigations in the diagnosis of sepsis. J R Coll Physicians Edinb. 2019;49:207-

8. Rello J, Valenzuela-Sánchez F, Ruiz-Rodriguez M, Moyano S. Sepsis: a review of advances in management. Adv Ther. 2017;34:2393-

9. Kitamura K, Kangawa K, Kawamoto M, Ichiki Y, Nakamura S, Matsuo H, et al. Adrenomedullin: a novel hypotensive peptide isolated from human pheochromocytoma. Biochem Biophys Res Commun. 1993;192:553-

10. Kato J, Kitamura K. Bench-to-bedside pharmacology of adrenomedullin. Eur J Pharmacol. 2015;764:140-

11. Ebara T, Miura K, Okumura M, Matsuura T, Kim S, Yukimura T, et al. Effect of adrenomedullin on renal hemodynamics and functions in dogs. Eur J Pharmacol. 1994;263:69-73.

12. Yamaguchi T, Baba K, Doi Y, Yano K. Effect of adrenomedullin on aldosterone secretion by dispersed rat adrenal zona glomerulosa cells. Life Sci. 1995;56:379-

13. Fukuhara M, Tsuchihashi T, Abe I, Fujishima M. Cardiovascular and neurohormonal effects of intravenous adrenomedullin in conscious rabbits. Am J Physiol. 1995;269:R1289-93.

14. Murphy TC, Samson WK. The novel vasoactive hormone, adrenomedullin, inhibits water drinking in the rat. Endocrinology. 1995;136:2459-

15. Bunton DC, Petrie MC, Hillier C, Johnston F, McMurray JJ. The clinical relevance of adrenomedullin: a promising profile? Pharmacol Ther. 2004;103:179-

16. Nishikimi T. Adrenomedullin in the kidney-renal physiological and pathophysiological roles. Curr Med Chem. 2007;14:1689-

Page $17 / 23$ 
17. Yuyun MF, Narayan HK, Ng LL. Prognostic significance of adrenomedullin in patients with heart failure and with myocardial infarction. Am J Cardiol. 2015;115:986-

18. Self WH, Storrow AB, Hartmann O, Barrett TW, Fermann GJ, Maisel AS, et al. Plasma bioactive adrenomedullin as a prognostic biomarker in acute heart failure. Am J Emerg Med. 2016;34:257-

19. Kim H, Hur M, Struck J, Bergmann A, Di Somma S. Circulating biologically active adrenomedullin predicts organ failure and mortality in sepsis. Ann Lab Med. 2019;39:454-

20. Mebazaa A, Geven C, Hollinger A, Wittebole X, Chousterman BG, Blet A, et al. Circulating adrenomedullin estimates survival and reversibility of organ failure in sepsis: the prospective observational multinational Adrenomedullin and Outcome in Sepsis and Septic Shock-1 (AdrenOSS-1) study. Crit Care. 2018;22:354.

21. Caironi P, Latini R, Struck J, Hartmann O, Bergmann A, Maggio G, et al. Circulating biologically active adrenomedullin (bio-ADM) predicts hemodynamic support requirement and mortality during sepsis. Chest. 2017;152:312-

22. Simon TP, Martin L, Deomming S, Humbs A, Bruells C, Kopp R, et al. Plasma adrenomedullin in critically ill patients with sepsis after major surgery: a pilot study. J Crit Care. 2016;38:68-

23. Helmy TA, Beshey BN. Prognostic role of adrenomedullin in sepsis. Int J Adv Res Biol Sci. 2016;3:136-

24. Marino R, Struck J, Maisel AS, Magrini L, Bergmann A, Di Somma S. Plasma adrenomedullin is associated with short-term mortality and vasopressor requirement in patients admitted with sepsis. Crit Care. 2014;18:R34.

25. Chen YX, Li CS. The predictive value of adrenomedullin for development of severe sepsis and septic shock in emergency department. Biomed Res Int. 2013;2013:960101.

26. Chen YX, Li CS. Prognostic value of adrenomedullin in septic patients in the ED. Am J Emerg Med. 2013;31:1017-

27. Bernal-Morell E, García-Villalba E, Vera MDC, Medina B, Martinez M, Callejo V, et al. Usefulness of midregional proadrenomedullin as a marker of organ damage and predictor of mortality in patients with sepsis. J Infect. 2018;76:249-57-

28. Önal U, Valenzuela-Sánchez F, Vandana KE, Rello J. Mid-regional pro-adrenomedullin (MR-proADM) as a biomarker for sepsis and septic shock: narrative review. Healthcare (Basel) 2018;6:E110.

29. Singer M, Deutschman CS, Seymour CW, Shankar-Hari M, Annane D, Bauer M, et al. The Third international consensus definitions for sepsis and septic shock (Sepsis-3). JAMA 2016;315:801-10.

30. Kitamura K, Sakata J, Kangawa K, Kojima M, Matsuo H, Eto T. Cloning and characterization of cDNA encoding a precursor for human adrenomedullin. Biochem Biophys Res Commun. 1993;194:720-

31. Kitamura K, Kato J, Kawamoto M, Tanaka M, Chino N, Kangawa K, et al. The intermediate form of glycine-extended adrenomedullin is the major circulating molecular form in human plasma. Biochem Biophys Res Commun. 1998;244:551-5.

32. Nomura I, Kato J, Tokashiki M, Kitamura K. Increased plasma levels of the mature and intermediate forms of adrenomedullin in obesity. Regul Pept. 2009;158:127-

33. Kawano S, Kawagoe Y, Kuwasako K, Shimamoto S, Igarashi K, Tokashiki M, el al. Gender-related alterations in plasma adrenomedullin level and its correlation with body weight gain. Endocr Connect. 2015;4:43-

34. Faul F, Erdfelder E, Buchner A, Lang AG. Statistical power analyses using G*Power 3.1: tests for correlation and regression analyses. Behav Res Methods. 2009;41:1149-

35. Bone RC, Balk RA, Cerra FB, Dellinger RP, Fein AM, Knaus WA, et al. Definitions for sepsis and organ failure and guidelines for the use of innovative therapies in sepsis. Crit Care Med. 1992;20:864-

36. Levy MM, Fink MP, Marshall JC, Abraham E, Angus D, Cook D, et al. 2001 SCCM/ESICM/ACCP/ATS/SIS International Sepsis Definitions Conference. Crit Care Med. 2003;31:1250-

37. Fang X, Wang Z, Yang J, Cai H, Yao Z, Li K, et al. Clinical evaluation of Sepsis-1 and Sepsis-3 in the ICU. Chest. 2018;153:1169-76:

Page $18 / 23$ 
38. Watanabe, K, Nishikimi T, Takamuro M, Yasuda K, Ishikawa Y, Tanabe S, et al. Two molecular forms of adrenomedullin in congenital heart disease. Pediatr Cardiol. 2003;24:559-

39. Yoshikawa D, Kawahara F, Okano N, Hiraoka H, Kadoi Y, Fujita N, et al. Increased plasma concentrations of the mature form of adrenomedullin during cardiac surgery and hepatosplanchnic hypoperfusion. Anesth Analg. 2003;97:663-

40. Cao, YN, Kitamura K, Ito K, Kato J, Hashida S, Morishita K, et al. Glycine-extended adrenomedullin exerts vasodilator effect through amidation in the rat aorta. Regul Pept. 2003;113:109-14.

41. Di Somma S, Magrini L, Travaglino F, Lalle I, Fiotti N, Cervellin G, et al. Opinion paper on innovative approach of biomarkers for infectious diseases and sepsis management in the emergency department. Clin Chem Lab Med. 2013;51:1167-

42. Weber J, Sachse J, Bergmann S, Sparwaßer A, Struck J, Bergmann A. Sandwich immunoassay for bioactive plasma adrenomedullin. J Appl Lab Med. 2017;2:222-

43. Nakamura Y, Ishikura H, Nishida T, Kawano Y, Yuge R, Ichiki R, et al. Usefulness of presepsin in the diagnosis of sepsis in patients with or without acute kidney injury. BMC Anesthesiol. 2014;14:88.

44. Nakamura Y, Hoshino K, Kiyomi F, Kawano Y, Mizunuma M, Tanaka J, et al. Comparison of accuracy of presepsin and procalcitonin concentrations in diagnosing sepsis in patients with and without acute kidney injury. Clin Chim Acta. 2019;490:200-

45. Bernal-Morell E, García-Villalba E, Vera MDC, Medina B, Martinez M, Callejo V, et al. Usefulness of midregional proadrenomedullin as a marker of organ damage and predictor of mortality in patients with sepsis. J Infect. 2018;76:249-

46. Wacker C, Prkno A, Brunkhorst FM, Schlattmann P. Procalcitonin as a diagnostic marker for sepsis: a systematic review and meta-analysis. Lancet Infect Dis. 2013;13:426-

47. Kondo Y, Umemura Y, Hayashida K, Hara Y, Aihara M, Yamakawa K. Diagnostic value of procalcitonin and presepsin for sepsis in critically ill adult patients: a systematic review and meta-analysis. J Intensive Care. 2019;7:22.

48. Li Q, Wang BS, Yang L, Peng C, Ma LB, Chai C. Assessment of adrenomedullin and proadrenomedullin as predictors of mortality in septic patients: a systematic review and meta-analysis. Med Intensiva. 2018;42:416-

49. Geven C, Kox M, Pickkers P. Adrenomedullin and adrenomedullin-targeted therapy as treatment strategies relevant for sepsis. Front Immunol 2018;9:292.

50. Geven C, Blet A, Kox M, Hartmann O, Scigalla P, Zimmermann J, et al. A double-blind, placebo-controlled, randomised, multicentre, proof-of-concept and dose-finding phase II clinical trial to investigate the safety, tolerability and efficacy of adrecizumab in patients with septic shock and elevated adrenomedullin concentration (AdrenOSS-2). BMJ Open. 2019;9:e024475.

\section{Figures}


Fig. 1

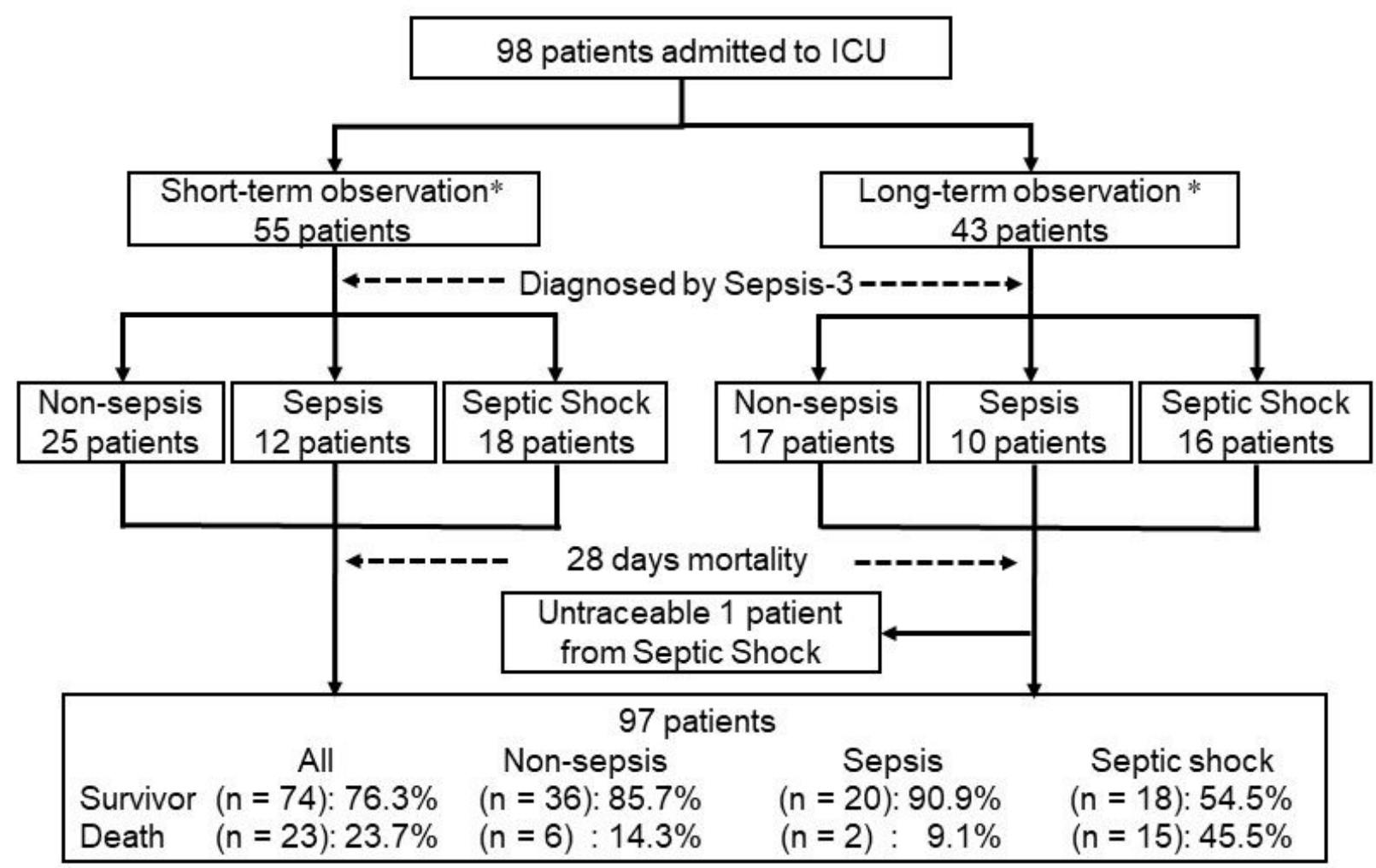

Figure 1

Flow chart of patient enrollment and classification by Sepsis-3 definitions. *Short-term observation was done within 24 hours of ICU admission. **Long-term observation was done within 7 days of ICU admission. We could not track the outcome of one septic shock patient in the long-term observational group. ICU, intensive care unit. 
Fig. 2
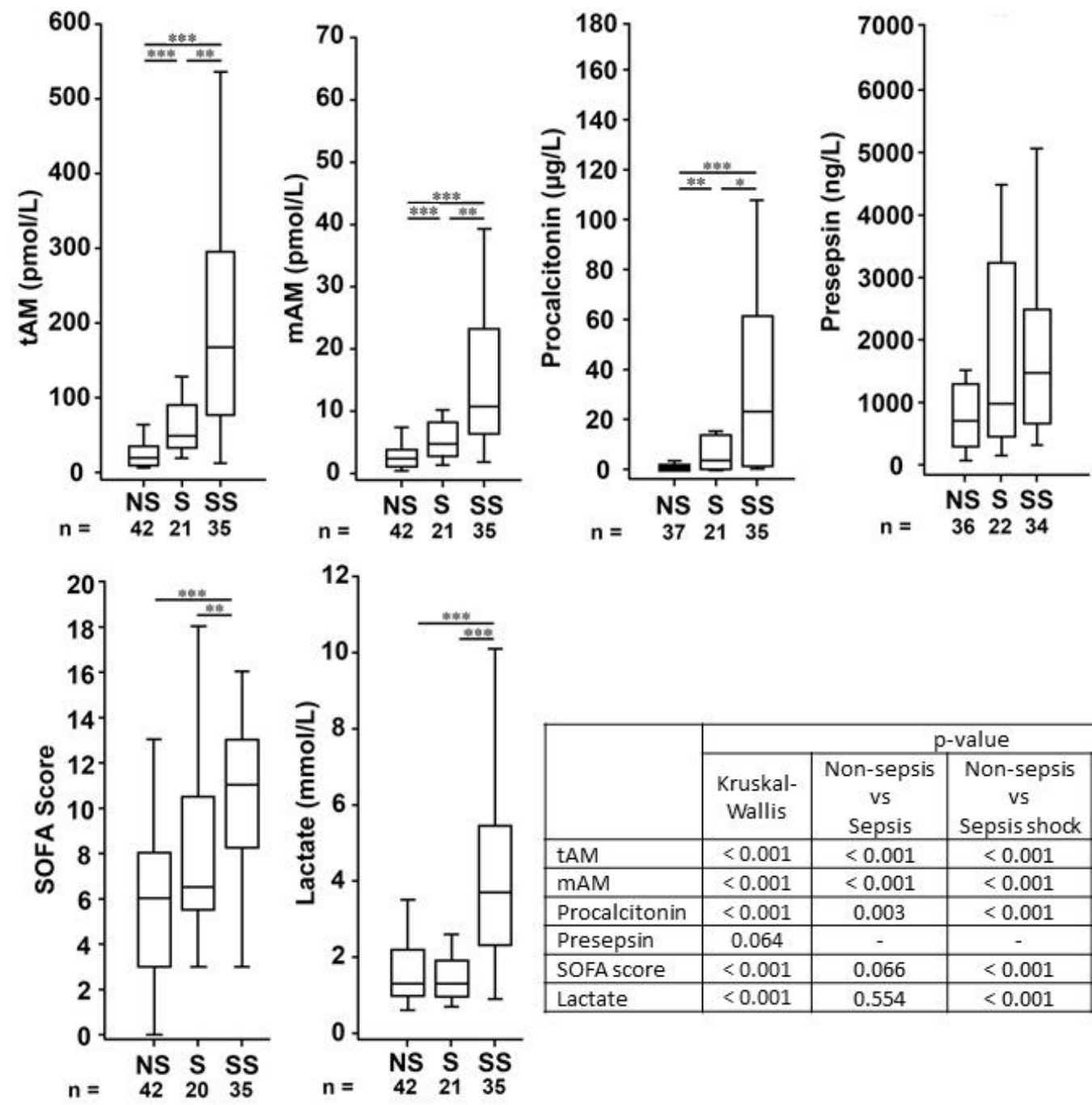

\begin{tabular}{|c|c|c|c|c|}
\hline & \multicolumn{4}{|c|}{ p-value } \\
\hline & $\begin{array}{c}\text { Kruskal- } \\
\text { Wallis }\end{array}$ & $\begin{array}{c}\text { Non-sepsis } \\
\text { vs } \\
\text { Sepsis }\end{array}$ & $\begin{array}{l}\text { Non-sepsis } \\
\text { vs } \\
\text { Sepsis shodk }\end{array}$ & $\begin{array}{c}\text { Sepsis } \\
\text { vs } \\
\text { Sepsis shock }\end{array}$ \\
\hline tAM & $<0.001$ & $<0.001$ & $<0.001$ & 0.002 \\
\hline $\mathrm{mAM}$ & $<0.001$ & $<0.001$ & $<0.001$ & 0.009 \\
\hline Procalcitonin & $<0.001$ & 0.003 & $<0.001$ & 0.016 \\
\hline Presepsin & 0.064 & - & - & - \\
\hline SOFA score & $<0.001$ & 0.066 & $<0.001$ & 0.002 \\
\hline Lactate & $<0.001$ & 0.554 & $<0.001$ & $<0.001$ \\
\hline
\end{tabular}

Figure 2

Comparative plasma expression of biomarkers and SOFA scores among non-sepsis, sepsis, and septic shock patients. Group comparisons were performed using Kruskal-Wallis and post-hoc Mann-Whitney U test. *p $<0.05,{ }^{\star \star} p<0.01,{ }^{\star \star \star} p<$ 0.001. NS, non-sepsis; S, sepsis; SS, septic shock; tAM, total adrenomedullin; mAM, mature adrenomedullin; SOFA, sequential organ failure assessment. 
Fig. 3 (A) Short-termed observation
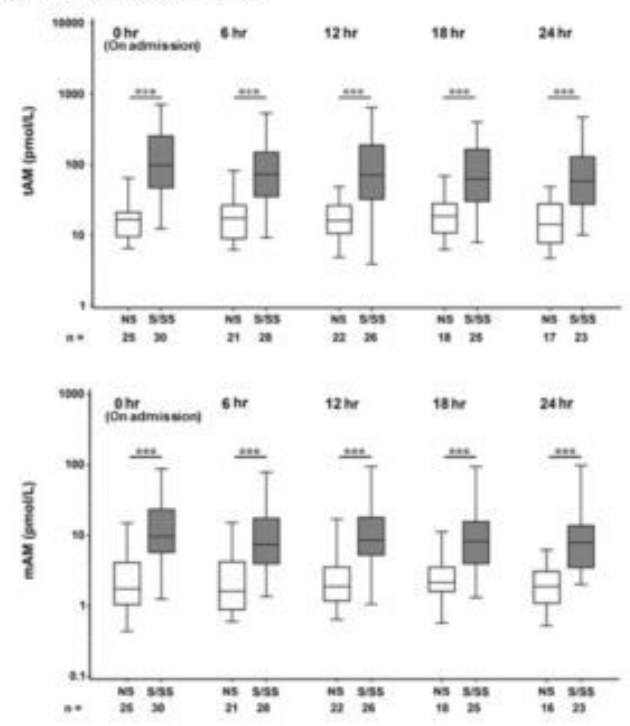

Fig. 3 (B) long-termed observation


\section{Figure 3}

Comparative plasma levels of tAM and mAM of non-sepsis and sepsis/septic shock patients. (a) Short-term observations were acquired within 24 hours of ICU admission. (b) Long-term observations were acquired within 7 days of ICU admission. Group comparisons were performed using the Mann-Whitney U test; ${ }^{\star} p<0.05,{ }^{\star *} p<0.01,{ }^{* \star} p<0.001$. NS, non-sepsis; S, sepsis; SS, septic shock; tAM, total adrenomedullin; mAM, mature adrenomedullin. 
Fig. 4

(A) Sepsis and septic shock

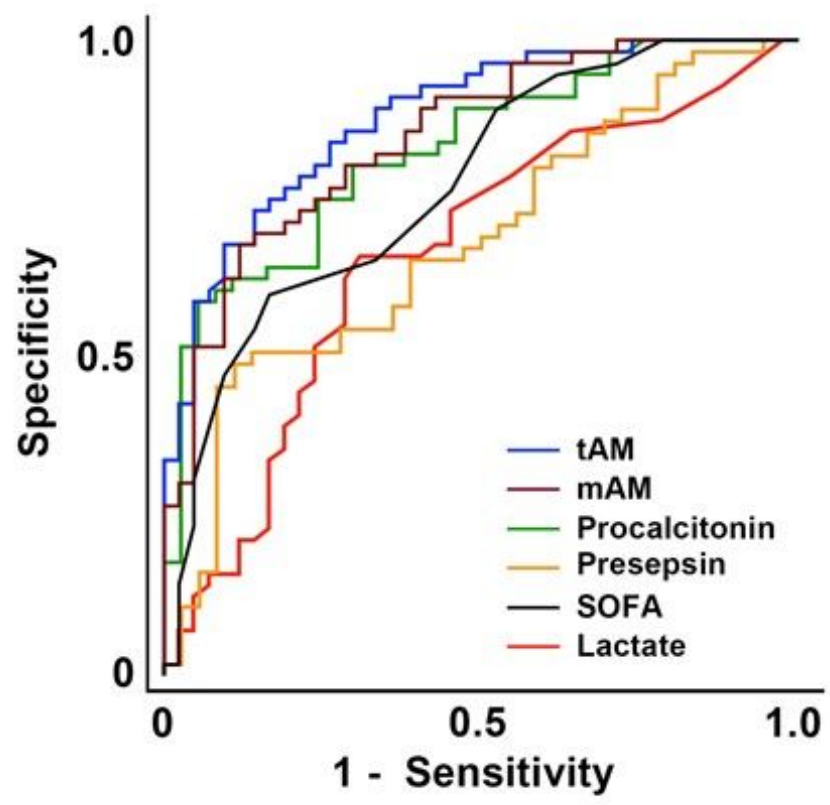

(B) Septic shock

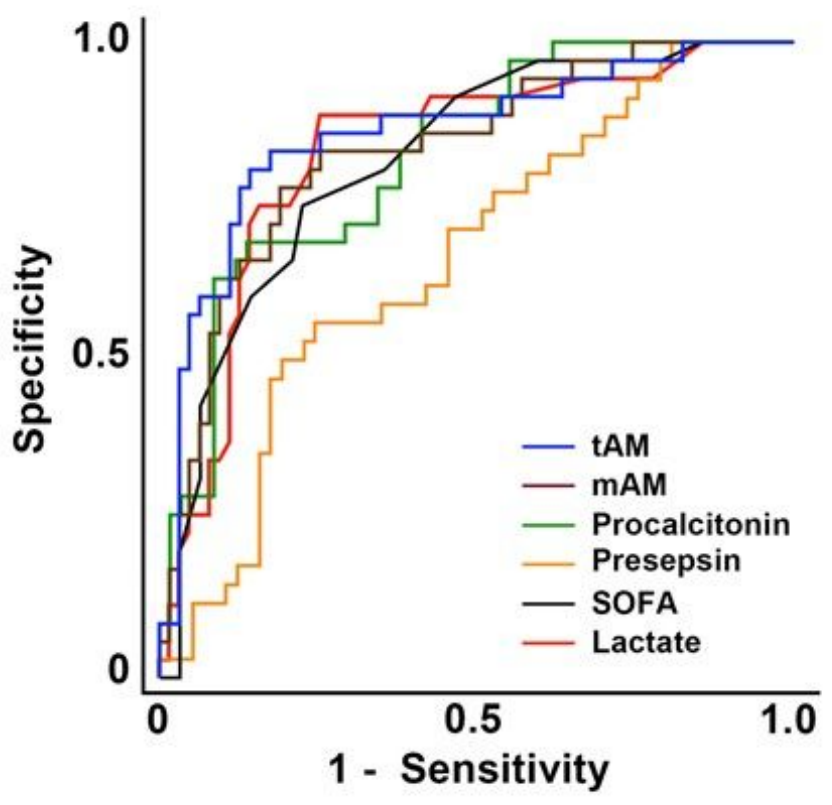

Figure 4

Receiver operating characteristics (ROC) analyses of biomarkers for the diagnosis/prognosis of sepsis and septic shock.

(a) ROC analyses of biomarkers for patients diagnosed with sepsis or septic shock as defined by Sepsis-3. (b) ROC analyses of biomarkers for patients with septic shock as defined by Sepsis-3. ROC analyses were performed by calculating the area under the curve (AUC). tAM, total adrenomedullin; mAM, mature adrenomedullin; SOFA, sequential organ failure assessment score. 